\title{
De la PAU a la EBAU: un análisis en el dominio de las Matemáticas Aplicadas a las Ciencias Sociales
}

\section{From the PAU to the EBAU: An analysis in the field of Mathematics applied to the Social Sciences}

\author{
Rosa Nortes Martínez-Artero ${ }^{1}$ \\ mrosa.nortes@um.es \\ Antonio de Pro Bueno \\ nono@um.es \\ Andrés Nortes ChecA \\ anortes@um.es \\ Universidad de Murcia, España
}

\section{Resumen:}

La EBAU (Evaluación del Bachillerato para el Acceso a la Universidad) ha sustituido a la PAU (Prueba de Acceso a la Universidad). Para conocer si ha sufrido modificaciones dicha prueba en el dominio de las Matemáticas Aplicadas a las Ciencias Sociales, se ha tomado una muestra de 138 alumnos examinados en la convocatoria de junio de 2018 en la Comunidad Autónoma de Murcia. Comparados sus resultados con estudios anteriores sobre la PAU se constata la continuidad de la prueba, con características semejantes y cuestiones análogas. Se repiten los errores y aunque disminuye el porcentaje de suspensos, se mantiene una nota media inferior a 5 . El $20 \%$ de las cuestiones fueron contestadas bien y el $25 \%$ obtuvieron una calificación de cero.

\begin{abstract}
:
In Spain the EBAU has replaced the PAU as the university access test. To know if the content of this test in the field of mathematics applied to social sciences has undergone changes, a sample of 138 students who took the exam in June 2018 in the Autonomous Community of Murcia was analyzed with a view to comparing results with those obtained in the PAU, as reported in previous studies: the continuity of the test, with similar characteristics and analogous issues, is confirmed. The errors are repeated and, although the fail percentage decreases, the average score continues to be lower than $5.20 \%$ of questions were answered correctly and $25 \%$ were responded incorrectly by all students.
\end{abstract}

1 Dirección para correspondencia (correspondence address):

Rosa Nortes Martínez-Artero. Facultad de Educación. Departamento de Didáctica de las Ciencias Matemáticas y Sociales. Universidad de Murcia. Campus de Espinardo, s/n. 30100 Espinardo (España). 
De la PAU a la EBAU: un análisis en el dominio de las Matemáticas Aplicadas a las Ciencias Sociales

Rosa Nortes Martínez-Artero, Antonio de Pro Bueno y Andrés Nortes Checa

\section{Palabras clave:}

Prueba de acceso a la universidad (PAU); evaluación del bachillerato para el acceso a la universidad (EBAU); matemáticas aplicadas a las ciencias sociales; calificación; exámenes de matemáticas.

\section{Key words:}

University access test (PAU); baccalaureate assessment for university access (EBAU); applied mathematics to social sciences; qualification; mathematics exams.

\section{Résumé:}

L'EBAU (Évaluation du Bachillerato pour I'Accès à I'Université) a remplacé le PAU (Épréuve d'Accès à l'Université). Afin de savoir si cette épreuve a subi des changements dans le domaine des Mathématiques Appliquées aux Sciences Sociales, un échantillon de 138 étudiants examinés lors de I'appel de juin 2018 dans la Communauté Autonome de Murcia a été pris. La comparaison de leurs résultats avec les études précédentes sur le PAU montre la continuité du test, avec des caractéristiques et des questions similaires. Les erreurs sont répétées et bien que le pourcentage d'échecs diminue, une note moyenne inférieure à 5 est maintenue. $20 \%$ des questions ont reçu une bonne réponse et $25 \%$ ont obtenu la note zéro.

\section{Mots clés:}

Examen d'entrée à l'université (PAU); évaluation du baccalauréat pour l'entrée à l'université (EBAU); mathématiques appliquées aux sciences sociales; notation; examens de mathématiques.

Fecha de recepción: 18-11-2019

Fecha de aceptación: 03-02-2020

\section{Introducción}

Las Pruebas de Acceso a la Universidad surgen por múltiples y variados motivos; entre ellos, la necesidad de establecer un mecanismo de prelación de estudiantes que quieren acceder a la universidad, sobre todo en las titulaciones más demandadas. Una de sus fortalezas sería que trata de homogeneizar los criterios de evaluación de diferentes centros y profesores. Y, entre sus debilidades, estaría la falta de especificidad de las pruebas para seleccionar a los estudiantes más adecuados en los Grados eminentemente profesionales.

Para el curso 2018-2019 la Orden PCI/12/2019 determina las características, el diseño y el contenido para acceso a la Universidad y las fechas máximas de realización y en su artículo 3 se especifica que "las pruebas versarán sobre las materias generales del bloque de asignaturas troncales de segundo curso de Bachillerato de la modalidad elegida para la prueba..." (p. 2678). Y en donde en Matemáticas Aplicadas a las Ciencias Sociales II (MACS) en la matriz de especificaciones se asigna 
al bloque de Procesos, métodos y actitudes matemáticas el 20\%, al de Números y álgebra el 25\%, al de Análisis el 25\% y al de Estadística y probabilidad el 30\%, estableciendo los correspondientes estándares de aprendizaje evaluables en cada uno de ellos.

\section{Antecedentes y Marco teórico}

Algunos de los estudios más recientes sobre las PAU son los siguientes:

Miralles y Deulofeu (2009), al analizar las razones que Ilevan a los alumnos a elegir una u otra opción en las pruebas de Matemáticas Aplicadas a las Ciencias Sociales II, concluyen que eligen problemas de enunciados con términos lingüísticos más sencillos, aunque sean más difíciles de resolver.

En Nortes y Nortes (2010), la muestra la constituyen las respuestas de 134 exámenes correspondientes a Matemáticas Aplicadas a las Ciencias Sociales II (MACS) de las Pruebas de Acceso a la Universidad (PAU) de septiembre de 2009. Se presentaban diez cuestiones repartidas en cinco bloques: números y álgebra; representación de una función; derivadas e integrales; probabilidad y una quinta destinada a intervalos de confianza y contraste de hipótesis. De cada bloque los alumnos debían elegir una cuestión y el porcentaje de elección más alto, el 91\%, fue el estudio de una curva. La nota media fue de 4.5 y la desviación típica de 2.2.

Rodríguez-Muñiz, Torres y Álvarez-Martino (2012), tras revisar los datos de 4731 alumnos asturianos de la PAU del año 2009, utilizan los resultados de 75 estudiantes elegidos al azar y analizan la asociación entre las notas medias individuales del Bachillerato y de la PAU, la calidad de la corrección de la prueba evaluando el impacto de los correctores y la capacidad para detectar diferencias significativas desde el punto de vista estadístico, entre otras. De esta manera, pretenden ofrecer una amplia información destinada a los diferentes usuarios que incluye la posibilidad de cotejar los resultados de los alumnos con el conjunto de estudiantes examinados.

Mallart (2014) analiza una muestra de 104 exámenes de Matemáticas de las Pruebas de Acceso a la Universidad de 2012 y estudia las resoluciones de los alumnos para detectar errores y poder incidir en dichas dificultades de aprendizaje. Llega a la conclusión que los alumnos asimilan los procedimientos mecánicos pero que carecen de creatividad a 
De la PAU a la EBAU: un análisis en el dominio de las Matemáticas Aplicadas a las

Rosa Nortes Martínez-Artero, Antonio de Pro Bueno y Andrés Nortes Checa

la hora de solucionar problemas geométricos y tienen poca precisión en las operaciones, añadiendo que hay una "ausencia de rigor y precisión en los cálculos efectuados" (p. 251).

Lorenzo, Argos, Hernández y Vera (2014) indicaban que las Pruebas de Acceso solo sirven para ubicar adecuadamente al alumnado, ordenarlo y darle prioridad a la elección por lo que proponen que sería más adecuado plantear un acceso a la universidad con una prueba específica para ingresar en estudios de límite de plazas.

Nortes, Nortes y Lozano (2015) utilizaron una muestra de 291 exámenes de primera evaluación y 141 exámenes presentados a la revisión en la PAU correspondiente a MACS de junio de 2014. Dos son los profesores que corrigen: por un lado, el profesor P1 tiene una muestra de 147 exámenes y las dos opciones A y B que las eligen 49 y 98 alumnos, respectivamente; por otro, el profesor P2 tiene 144 exámenes de los que 57 son de la opción A y 87 de la opción B. La media del profesor P1 es 3.95 (Opción A=4.83 y Opción B=3.51) y la del profesor P2 es 4.07 (Opción $A=4.29$ y Opción $B=3.92$ ), no habiendo diferencias significativas entre las correcciones de los dos profesores.

Mengual, Albarracín, Muñoz-Escolano, Oller-Marcén y Gorgorió (2019) diseñan criterios para reducir la variabilidad en la calificación de exámenes de Matemáticas en las PAU otorgadas por distintos correctores. Para ello distinguen entre tareas principales y tareas auxiliares, según constituyan el objetivo principal de la calificación o que aparezcan en el proceso de resolución del problema, estableciendo tareas auxiliares específicas y tareas auxiliares generales en donde el conjunto de errores no podrá penalizarse con más de $2 / 3$ de la calificación otorgada a la respuesta correcta y con más de $1 / 3$, respectivamente.

Rodríguez-Muñiz, Díaz, Mier y Alonso (2016) analizan el tipo de ejercicios y problemas que se proponen en las PAU en la asignatura MACS y su relación con los currículos oficiales al tiempo que estudian la dualidad entre los currículos oficiales y los reales en Andalucía, Asturias, Madrid y País Vasco. Para ello utilizan datos de 154 exámenes y 628 ejercicios PAU desde 2010 a 2014. Además, a 51 profesores de secundaria de $2 .^{\circ}$ de bachillerato que imparten la asignatura MACS, se les aplicó un cuestionario, en el que alrededor del $50 \%$ reconoce que, si las PAU no existieran, su enseñanza estaría más cerca del plan de estudios e intentarían enseñar la totalidad del programa y que cuando hay falta de tiempo, se eligen los contenidos de las PAU. 
En este mismo sentido se pronuncian Ordóñez y Contreras (2011) que consideran que las PAU tienen una gran influencia en la enseñanza y aprendizaje en $2 .^{\circ}$ de bachillerato y pueden llegar a ser verdaderas restricciones institucionales. A esta conclusión llegan tras analizar el caso de la integral definida y los resultados de la revisión de los manuales y apuntes de clase y observar una gran coincidencia con las mencionadas pruebas.

Boal, Bueno, Leris y Sein-Echaluce (2008), tras analizar las habilidades matemáticas evaluadas en las PAU al finalizar la educación preuniversitaria, "han detectado un enfoque superficial del aprendizaje de las matemáticas de bachillerato: aprender a reproducir la información para cumplir con los requisitos de la PAU" (p. 21).

Sin embargo, Ruiz de Gauna, Dávila, Etxeberría y Sarasua (2013) indicaron que la prueba condicionaba la metodología de enseñanza; en concreto, señalan que, en Matemáticas Aplicadas a las Ciencias Sociales II en $2 .^{\circ}$ de bachillerato se trabaja para preparar los ejercicios y problemas de selectividad, pero piensan que la prueba no condiciona el objetivo último del proceso de enseñanza-aprendizaje porque por encima de la preparación del examen está que los alumnos aprendan Matemáticas.

La EBAU ha venido a sustituir a la PAU como prueba de acceso a la universidad española. Sus características vienen reguladas en la Orden ECD/42/2018 mediante trece artículos, siendo el 5) en el que se establece "la concreción de los estándares de aprendizaje evaluables asociados a cada uno de los bloques de contenidos que darán cuerpo al proceso de evaluación" (p. 9759). Esta Orden es el desarrollo del Real Decretoley 5/2016, en el que se decía que, hasta la entrada en vigor de la normativa resultante del Pacto de Estado Social y Político por la Educación, en el caso del Bachillerato "se realizará una prueba de características semejantes a la hasta ahora vigente Prueba de Acceso a la Universidad y válida a los solos efectos de acceso a la universidad" (p. 86169). Como el ansiado Pacto no ha llegado, la Orden PCl/12/2019 determina las características, el diseño y el contenido para acceso a la Universidad para el curso 2018-2019.

Los estudios anteriores se realizaron con las PAU. Desde que entraron en vigor las EBAU, los estudios realizados hasta ahora son inexistentes. De ahí que, habiendo realizado estudios (Nortes y Nortes, 2010, Nortes et al., 2015) en las PAU, se quiera realizar un estudio semejante en las EBAU, que permita contrastar los resultados en ambas pruebas. 
De la PAU a la EBAU: un análisis en el dominio de las Matemáticas Aplicadas a las Ciencias Sociales

Rosa Nortes Martínez-Artero, Antonio de Pro Bueno y Andrés Nortes Checa

\section{Objetivo}

El objetivo del presente estudio es analizar los resultados de la prueba EBAU de MACS de los exámenes corregidos por un profesor, clasificando la forma de resolución de las cuestiones, detectando los errores más significativos y comparando los resultados con otras pruebas de esta materia de PAU.

\section{Metodología}

\section{Participantes}

La base documental del presente trabajo son las respuestas de 138 estudiantes de distintos centros escolares de la Comunidad Autónoma de Murcia que se presentaron a las pruebas de EBAU en la convocatoria de junio (11.06.2018).

\section{Instrumento}

La estructura de la prueba EBAU de la Comunidad Autónoma de Murcia de la materia Matemáticas Aplicadas a las Ciencias Sociales II (MACS), consiste en dos opciones a elegir una, con cinco cuestiones para cada opción y duración de 90 minutos. Las cinco cuestiones corresponden a: Parte 1 (30-35\%), que son del bloque 1 (Procesos, métodos y actitudes) y bloque 2 (Números y Álgebra); Parte 2 (30-40\%), que son de los bloques 1 y 3 (Análisis) y Parte 3 (30-40\%), que son de los bloques 1 y 4 (Probabilidad y Estadística). De ahí que una cuestión sea de la parte 1 con puntuación 3, dos cuestiones de la parte 2 con puntuación 3.5, y dos cuestiones de la parte 3 con puntuación 3.5. Los enunciados y la puntuación de cada cuestión se recogen en las Figuras 1 (Opción A) y 2 (Opción B). 
De la PAU a la EBAU: un análisis en el dominio de las Matemáticas Aplicadas a las

\section{OPCIÓN A}

Cuestión A1. [3 puntos]

Discutir el siguiente sistema en función del parámetro a:

$$
\begin{gathered}
x+2 y-z=6 \\
\begin{array}{l}
y+z=1 \\
a x+y-2 z=4 \\
\text { Resolverlo para } a=2
\end{array} \quad \text { (2,5 puntos) } \\
\hline
\end{gathered}
$$

Cuestión A2. [2 puntos]

Una empresa fabrica un determinado producto que vende al precio unitario de 15 euros. La función de costes, que representa el coste (en unidades monetarias) en función del número de unidades de producto, es $\mathrm{C}(\mathrm{x})=2 \mathrm{x}^{2}$ $45 x+300$, donde $x$ es el número de unidades del producto. Hallar el número de unidades que ha de vender para obtener el máximo beneficio sabiendo que el beneficio es igual al ingreso total obtenido por la venta menos los costes. Calcular el beneficio máximo.

Cuestión A3. [1,5 puntos]

Hallar el área del recinto acotado limitado por la gráfica de la función $y=x^{2}-$ $x-2$, el eje OX y las rectas $x=-2$ y $x=2$. Hacer la representación gráfica de dicha área.

\section{Cuestión A4. [2 puntos]}

El examen de una asignatura consta de tres pruebas. La primera prueba es superada por el $80 \%$ de los alumnos que la realizan. Esta prueba es eliminatoria, por lo que si no se supera no se pueden realizar las otras, y se suspende la asignatura. La segunda prueba tiene dos convocatorias en las que puede superarse, la ordinaria y la extraordinaria (para alumnos que no la hayan superado en la ordinaria). Superan esta prueba el $35 \%$ de los alumnos en la convocatoria ordinaria y el $50 \%$ de los alumnos que se presentan a la extraordinaria. La tercera prueba solo pueden realizarla los alumnos que tienen las otras dos pruebas superadas, y la supera el $75 \%$ de los alumnos presentados.

a) Calcular la probabilidad de superar las dos primeras pruebas. (1,5 puntos)

b) Si el requisito para aprobar la asignatura es que se superen las tres pruebas, hallar la probabilidad de aprobar la asignatura. (0,5 puntos)

Cuestión A5. [1,5 puntos]

En una muestra aleatoria de tamaño 200 de árboles de una población se ha obtenido que 45 tienen una plaga. Hallar el intervalo de confianza al $90 \%$ para la proporción de árboles que tienen la plaga.

Figura 1. Prueba EBAU de MACS (Opción A). 
De la PAU a la EBAU: un análisis en el dominio de las Matemáticas Aplicadas a las Ciencias Sociales

Rosa Nortes Martínez-Artero, Antonio de Pro Bueno y Andrés Nortes Checa

\section{OPCIÓN B}

Cuestión B1. [3 puntos]

Una fábrica produce dos modelos de bolsos, tipo A y tipo B. Cada bolso tipo $\mathrm{A}$ requiere $5 \mathrm{~m}^{2}$ de piel y 5 horas de trabajo y cada bolso del modelo $B$ requiere $5 \mathrm{~m}^{2}$ de piel y 10 horas de trabajo. Dispone de $200 \mathrm{~m}^{2}$ de piel y 225 horas de trabajo. Además, requiere producir mayor o igual número de bolsos tipo A que B. El beneficio obtenido es de 50 euros por cada bolso tipo A y 80 euros por cada bolso tipo B. Hallar el número de bolsos que debe fabricar de cada tipo para obtener el máximo beneficio. Calcular dicho beneficio máximo.

\section{Cuestión B2. [2 puntos]}

Hallar las derivadas de las siguientes funciones:

$$
\begin{aligned}
& f(x)=\frac{-1}{\sqrt[5]{5 x+1}}(0,75 \text { puntos }) \\
& g(x)=x^{2} \ln x^{2}(0,75 \text { puntos }) \\
& h(x)=e^{-3 x+x 2}(0,5 \text { puntos })
\end{aligned}
$$

Cuestión B3. [1,5 puntos]

Dadas las funciones $f(x)=e^{x}$ y $g(x)=x^{3}-2 x+1$, cuyas gráficas aparecen en la figura:

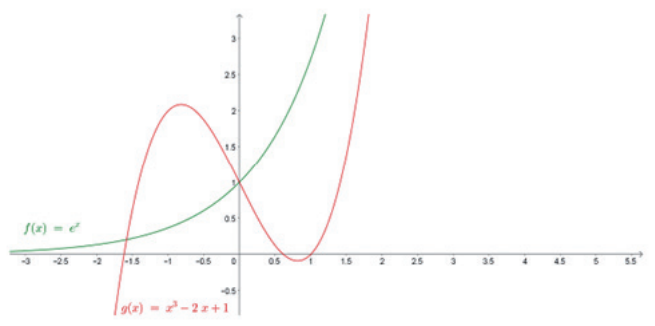

Hallar el área del recinto acotado por las dos gráficas y las rectas $\mathrm{x}=-1$ y $\mathrm{x}=0$. Cuestión B4. [2 puntos]

La probabilidad de que un autobús llegue con retraso a una parada es 0,2 . Si pasa cuatro veces a lo largo del día por la parada, calcular la probabilidad de que:

a) No llegue con retraso ninguna de las veces.

b) Llegue con retraso al menos una vez.

c) Al menos tres veces llegue con retraso.

d) Llegue con retraso exactamente dos veces consecutivas.

Cuestión B5. [1,5 puntos]

La altura para una determinada población sigue una distribución normal con una desviación típica conocida (sigma). Para hallar un intervalo de confianza para la media de la población se ha tomado una muestra aleatoria simple de 100 individuos, obteniéndose una altura media de $145 \mathrm{~cm}$. Si el intervalo de confianza con un nivel de significación 0,05 construido a partir de los datos anteriores es $(135,2,154,8)$, hallar el valor de sigma.

Figura 2. Prueba EBAU de MACS (Opción B). 
Como criterios generales señalados por el coordinador de la materia cada error trivial se penaliza con 0.1 puntos y cada error de cálculo no trivial con 0.2 puntos. Para cada cuestión se indican unos criterios específicos en donde se detallan las distintas formas de resolución y la puntuación de cada paso y que en una reunión previa a la corrección de la prueba fue analizada, matizada y consensuada por todos los correctores.

Dentro de los estándares de aprendizaje evaluables recogidos en la matriz de especificaciones de la Orden PCl/12/2019, A1 y B2 se sitúan en "Analiza y comprende el enunciado a resolver (datos, relaciones entre los datos, condiciones, conocimientos matemáticos necesarios, etc.)" (Procesos, métodos y actitudes en matemáticas); B1 en "Aplica las técnicas gráficas de programación lineal bidimensional para resolver problemas de optimización de funciones lineales que están sujetas a restricciones e interpreta los resultados obtenidos en el contexto del problema" (Números y álgebra); A2 en "Plantea problemas de optimización sobre fenómenos relacionados con las ciencias sociales, los resuelve e interpreta el resultado obtenido dentro del contexto" (Números y álgebra); A3 y B3 en "Aplica el concepto de integral definida para calcular el área de recintos planos delimitados por una o dos curvas" (Análisis); A4 y B4 en "Resuelve una situación relacionada con la toma de decisiones en condiciones de incertidumbre en función de la probabilidad de las distintas opciones" (Estadística y probabilidad) y A5 y B5 en "Relaciona el error y la confianza de un intervalo de confianza con el tamaño muestral y calcula cada uno de estos tres elementos conocidos los otros dos y lo aplica en situaciones reales" (Estadística y probabilidad). Estos estándares de aprendizaje evaluables están recogidos en el Decreto 221/2015 del currículo de Bachillerato en la Región de Murcia, por lo que la prueba analizada se adecua a la legislación que regula la EBAU.

\section{Estrategias de análisis}

Ruíz de Gauna (2010) establece una clasificación dicotómica de dificultad para los ejercicios de una Prueba de Acceso a la Universidad: fácil (F) y difícil (D). A continuación en la tabla 1 se presentan las características de las dos tipologías y en la tabla 2 la clasificación de la prueba analizada consensuada por los autores de la presente investigación. 
De la PAU a la EBAU: un análisis en el dominio de las Matemáticas Aplicadas a las Ciencias Sociales

Rosa Nortes Martínez-Artero, Antonio de Pro Bueno y Andrés Nortes Checa

Tabla 1

Características de la dificultad de una cuestión

\begin{tabular}{ll}
\hline Fácil & Difícil \\
\hline $\begin{array}{l}\text { 1. La comprensión del texto del ejer- } \\
\text { cicio es sencilla. }\end{array}$ & $\begin{array}{l}\text { 1. La comprensión del texto del ejer- } \\
\text { cicio no es sencilla. }\end{array}$ \\
$\begin{array}{ll}\text { 2. El ejercicio es identificable con sus } \\
\text { técnicas de resolución. }\end{array}$ & $\begin{array}{l}\text { 2ácilmente identificables. } \\
\text { 3. La resolución es directa. }\end{array}$ \\
$\begin{array}{ll}\text { 3. Para su resolución se requiere de } \\
\text { soluciones intermedias. }\end{array}$ \\
$\begin{array}{ll}\text { los largos y complicados. } & \text { 4. Los cálculos a que da lugar pueden } \\
\text { 5. El problema no contiene paráme- } & \text { 5. El problema presenta un cierto } \\
\text { tros de los cuales dependa la solu- } & \text { aspecto teórico. } \\
\text { ción. } & \end{array}$
\end{tabular}

Tabla 2

Clasificación de la prueba analizada

\begin{tabular}{cccccccccc}
\hline \multicolumn{4}{c}{ Opción A } & Opción B \\
\hline A1 & A2 & A3 & A4 & A5 & B1 & B2 & B3 & B4 & B5 \\
D & D & F & D & F & F & D & D & F & F \\
\hline
\end{tabular}

\section{Resultados}

Se analizan los resultados globales, de las cuestiones de cada bloque, la forma de resolución de los problemas y los errores más significativos.

\section{Resultados generales de la muestra}

De los 138 alumnos evaluados, 102 eligen la opción A y 36 la opción B. Una vez corregidos los exámenes, se ha obtenido por opción media, mediana, moda y desviación típica, siendo sus resultados los indicados en la tabla 3.

Tabla 3

Estadísticos de la muestra

\begin{tabular}{lccccc}
\hline & $\mathrm{N}$ & Media & Mediana & Moda & D.T. \\
\hline Opción A & 102 & 4.04 & 3.75 & 6.30 & 2.40 \\
Opción B & 36 & 5.13 & 5.55 & 3.10 & 2.21 \\
Total & 138 & 4.32 & 4.10 & 6.30 & 2.39 \\
\hline
\end{tabular}


De la PAU a la EBAU: un análisis en el dominio de las Matemáticas Aplicadas a las

La evaluación cualitativa por suspenso, aprobado, notable y sobresaliente se presenta en la tabla 4.

Tabla 4

Porcentajes evaluación cualitativa

\begin{tabular}{lcccc}
\hline & Suspenso & Aprobado & Notable & Sobresaliente \\
\hline Opción A & $62.75 \%$ & $24.51 \%$ & $8.82 \%$ & $3.92 \%$ \\
Opción B & $44.44 \%$ & $30.56 \%$ & $25.0 \%$ & $0 \%$ \\
TOTAL & $57.97 \%$ & $26.09 \%$ & $13.04 \%$ & $2.90 \%$ \\
\hline
\end{tabular}

A la vista de los valores, podemos decir:

- Más de la mitad de los alumnos que eligen la opción A suspenden, dos de cada tres aproximadamente, mientras que los que eligen la opción B uno de cada cuatro saca notable.

- En la opción B ningún alumno obtiene la calificación de Sobresaliente, pero más del 50\% aprueban.

- El $42 \%$ de los exámenes tienen nota igual o superior a 5.

- De las 690 cuestiones analizadas, el 20\% fueron contestadas bien, mientras que el $25 \%$ obtuvieron calificación de cero.

Los alumnos que se presentaron a la prueba EBAU de junio de 2018 fueron 2394, de los que 1035 aprobaron, el 43.2\%, y la nota media es de 4.52 , que supone dos décimas de diferencia en la media y un $1.2 \%$ en porcentaje de aprobados respecto a los resultados que presenta la muestra analizada.

\section{Resultados de las cuestiones de cada opción}

Se presentan dos tablas, tabla 5 y tabla 6, con los resultados más representativos de cada opción en donde las columnas son: valoración de la cuestión (Valor), porcentaje de aprobados (\% Apr.), porcentaje alumnos con calificación máxima (\% CM), porcentaje alumnos con cero (\% C0), Media (Med.), desviación típica (DT) y dificultad de la prueba (DP). De los 138 alumnos participantes, 102 alumnos respondieron a la Opción A y 36 a la opción B. 
De la PAU a la EBAU: un análisis en el dominio de las Matemáticas Aplicadas a las Ciencias Sociales

Rosa Nortes Martínez-Artero, Antonio de Pro Bueno y Andrés Nortes Checa

Tabla 5

Resultados Opción A

\begin{tabular}{lccccccc}
\hline & Valor & $\%$ Apr. & $\%$ CM & $\%$ C0 & Med. & DT & DP \\
\hline Cuestión A1 & 3 & 37.25 & 11.76 & 13.73 & 1.21 & 1.01 & Difícil \\
Cuestión A2 & 2 & 34.31 & 18.62 & 60.78 & 0.65 & 0.87 & Difícil \\
Cuestión A3 & 1.5 & 43.13 & 10.78 & 36.27 & 0.61 & 0.56 & Fácil \\
Cuestión A4 & 2 & 15.68 & 13.73 & 33.33 & 0.50 & 0.67 & Difícil \\
Cuestión A5 & 1.5 & 69.61 & 42.16 & 6.86 & 1.06 & 0.53 & Fácil \\
\hline
\end{tabular}

Tabla 6

Resultados Opción B

\begin{tabular}{lccccccc}
\hline & Valor & $\%$ Apr. & $\%$ CM & C0 & Med. & DT & DP \\
\hline Cuestión B1 & 3 & 55.56 & 25,0 & 2.78 & 1.73 & 0.95 & Fácil \\
Cuestión B2 & 2 & 38.89 & 8.33 & 16.67 & 0.72 & 0.66 & Difícil \\
Cuestión B3 & 1.5 & 25.0 & 11.11 & 27.78 & 0.67 & 0.53 & Difícil \\
Cuestión B4 & 2 & 52.78 & 25.0 & 30.56 & 1.02 & 0.84 & Fácil \\
Cuestión B5 & 1.5 & 50.0 & 44.44 & 16.67 & 0.98 & 0.58 & Fácil \\
\hline
\end{tabular}

Pasadas las medias a una calificación sobre 10, se presentan en la tabla 7.

Tabla 7

Medias por cuestión sobre 10

\begin{tabular}{lccccc}
\hline & Cuestión 1 & Cuestión 2 & Cuestión 3 & Cuestión 4 & Cuestión 5 \\
\hline Opción A & 4.03 & 3.25 & 4.07 & 2.50 & 7.07 \\
\hline Opción B & 5.77 & 3.60 & 4.47 & 5.20 & 6.53 \\
\hline Media ponderada & 4.48 & 3.34 & 4.17 & 3.28 & 6.93 \\
\hline
\end{tabular}

\section{Forma de resolución de las cuestiones}

Cuestión A1 (3 puntos). Este problema ha sido resuelto por dos procedimientos: Gauss (67 alumnos) y Cramer (30) y en blanco (5), de los que 1 y 11 obtienen la máxima calificación, que supone el 1\% y el 30.56\% de éxito. La media de los que lo hicieron por Cramer es de 1.97 y el porcentaje de aprobados del $70 \%$. Ningún alumno resuelve la segunda parte del sistema de tres ecuaciones con tres incógnitas por sustitución de una incógnita en las otras dos ecuaciones.

Cuestión B1 (3 puntos). La evaluación de este problema va desde 0.15 por poner una inecuación o la función a maximizar hasta los tres 
puntos por tenerlo completo. Comparando los resultados con los de la cuestión A1, ambas de álgebra, la media es de 1.73 frente a 1.21.

Cuestión A2 (2 puntos). Se parte de la función beneficio B(x) comprobando mediante la derivada segunda en el punto obtenido si alcanza un máximo o bien observando que el valor $B^{\prime}(x)$ es mayor que cero para $x$ inferior al valor encontrado y que $\mathrm{B}^{\prime}(\mathrm{x})$ es menor que cero para $\mathrm{x}$ mayor que el valor encontrado. Por este procedimiento lo hacen 13 alumnos y el resto por el indicado en primer lugar.

Cuestión B2 (2 puntos). Es un ejercicio de derivar tres funciones inmediatas y sin embargo no lo aprobará el 50\%, aun cuando en el enunciado no se pedía que se simplificara. La primera derivada en donde aparece una raíz de índice 5 solo fue resuelta bien por seis alumnos, el $16.7 \%$, mientras que 24 obtuvieron cero, el $66.67 \%$ y tres no contestaron, el $8.33 \%$. El resto hizo algo.

Cuestión A3 (1.5 puntos). Se pedía en primer lugar hacer la representación gráfica y hay alumnos que lo resuelven bien sin hacerla por lo que no pueden tener la puntuación máxima, siendo 17 los que lo hacen así; también es importante indicar que el $36.27 \%$ obtiene cero y el $10.78 \%$ obtiene la calificación máxima. Lo resuelven utilizando la función primitiva y la regla de Barrow.

Cuestión B3 (1.5 puntos). Las dos funciones dadas están representadas por sus gráficas correspondientes y están acotadas por dos rectas. Hay 10 alumnos que obtienen la puntuación máxima y 10 que obtienen la mínima, el $27.78 \%$ en cada caso. Se utiliza como procedimiento de resolución la obtención de la primitiva y aplicación de la regla de Barrow.

Cuestión A4 (2 puntos). Por diagrama de árbol lo hacen 3 de cada 4 alumnos, mientras que 1 de cada 4 lo hace sin diagrama de árbol. De los primeros 12 lo tienen bien y 21 tienen cero y de los segundos 2 lo tienen bien y 9 tienen cero, con lo que el porcentaje de respuestas con puntuación máxima es el doble utilizando diagrama de árbol que no utilizándolo.

Cuestión B4 (2 puntos). Utilizan diagrama de árbol 20 alumnos obteniendo 6 de ellos puntuación máxima de dos puntos, mientras que sin diagrama de árbol lo hacen 13 alumnos y 3 lo tienen bien, con porcentajes de $30 \%$ y $23.08 \%$, respectivamente.

Cuestión A5 (1.5 puntos). En este intervalo de confianza solo 7 alumnos tienen cero y 2 no contestan, siendo 42 los que tienen la puntuación 
De la PAU a la EBAU: un análisis en el dominio de las Matemáticas Aplicadas a las Ciencias Sociales

Rosa Nortes Martínez-Artero, Antonio de Pro Bueno y Andrés Nortes Checa

máxima, el $42.16 \%$ al tratarse de un ejercicio en donde poniendo bien la expresión y sustituyendo los datos del enunciado era inmediato.

Cuestión B5 (1.5 puntos). En este caso conocida la expresión del intervalo de confianza, se pedía obtener el valor de la desviación típica desconocida, por lo que era inmediato. Hay 6 alumnos con cero y 16 con la calificación máxima, lo que supone el $16.67 \%$ y el $44.44 \%$, respectivamente. Ningún alumno comprobó si el valor obtenido para sigma era correcto sustituyéndolo en el otro extremo del intervalo.

\section{Errores más significativos}

Se indica en cada cuestión los errores más reiterativos y, en algunos casos, la frecuencia.

Cuestión A1:

- En este problema el principal error al aplicar el método de Gauss es en la eliminación de incógnitas para llegar a la discusión en función del parámetro "a" al sumar filas o columnas. Un error que se repite es que hay alumnos que llegan a tener " $-3+3 a=0$ " y en la ampliada " $3-4 a=0$ ", tomando como dos valores $a=1$ y $a=3 / 4$ para la discusión.

- Los que aplican Cramer el principal error es la equivocación del determinante igualado a cero obteniendo valores distintos para " $a$ " que no son $\mathrm{a}=1$.

Cuestión B1:

- No señalan la restricción $x \geq y$, no la representan ni obtienen el punto de corte con otra recta.

- Representan mal las rectas.

- Dibujan mal la región factible.

- Se equivocan en algunos de los vértices.

- Hay equivocaciones en la función, como poner $f(x, y)=200 x+225 y$.

Cuestión A2:

- El primer error es que 36 alumnos, el 35.29\%, toman como función de beneficio la función de costes, Ilegando a obtener que el número de unidades es de 11.25, número decimal, que supone otro error al seguir adelante con el problema.

- El segundo es el que se comete con el signo menos delante de un 
paréntesis que hace cambiar de signo solo el primer término del paréntesis. Este error lo cometen 5 alumnos.

- Otros errores que cometen al poner la función de beneficios.

- Los hay que hacen directamente $\mathrm{B}(\mathrm{x})=0$ obteniendo dos soluciones 6.25 y 23.75 o 6.5 y 16 .

\section{Cuestión B2:}

- En la derivada de $f(x)$ no saben relacionar raíz y potencia a la hora de resolverla.

- En la derivada de la función $\mathrm{g}(\mathrm{x})$ desconocen la derivada del logaritmo neperiano.

- En la derivada de $h(x)$ no ponen el paréntesis en la derivada del exponente $(-3+2 x)$.

Cuestión A3:

- Dibujan mal la gráfica; lo hacen 53 alumnos, el 51.96\%.

- Integran directamente entre -2 y +2 , sin tener en cuenta la gráfica y los puntos de corte con el eje OX. Lo hacen 31 alumnos, el $30.39 \%$, siendo varios los que cometen los dos errores.

\section{Cuestión B3:}

- No observan que en el dibujo del recinto acotado $g(x)$ está por encima de $f(x)$ y que en el planteamiento es $g(x)-f(x)$. La mayoría lo pone al revés obteniendo respuestas muy diversas. Este error lo comete uno de cada dos alumnos.

- Un segundo error generalizado es no poner " $\mathrm{dx}$ " cuando van a integrar.

- Un tercer error son los signos cuando se aplica Barrow.

Cuestión A4:

- Hay un error que cometen 36 alumnos, el 35.29\%, consistente en calcular el apartado a) sin tener en cuenta que en la convocatoria extraordinaria supone haber suspendido la ordinaria y que luego arrastran al apartado b), que lo hacen 22, el 21.57\%.

- Otro error reiterado, aunque por menos alumnos, el 7.8\%, es considerar a) $\mathrm{P}=0.80 \times 0.35=0.28$ y b) $\mathrm{P}=0.28 \times 0.75=0.21$.

- Hay errores como dar valores de la probabilidad números superiores a 1 . 
De la PAU a la EBAU: un análisis en el dominio de las Matemáticas Aplicadas a las Ciencias Sociales

Rosa Nortes Martínez-Artero, Antonio de Pro Bueno y Andrés Nortes Checa

- Un error repetido por muchos alumnos ha sido el utilizar calculadoras que no conocían porque, al hacer una operación con decimales, ésta les daba el resultado en forma de fracción y desconocían el paso a forma decimal.

Cuestión B4:

- Hay alumnos que el apartado a) lo contestan poniendo $\mathrm{P}=1$ $0.8=0.2$ o $\mathrm{P}=1-0.2=0.8$.

- Un alumno calcula las combinaciones de R y R' considerando las cuatro paradas obteniendo 14 casos posibles.

- En el apartado c) "al menos" un alumno lo confunde con una proporcionalidad condicionada.

Cuestión A5:

- Uno de los errores es el valor de z, obteniendo 0.8289; 0.828; 1 ; 0.40800 o 1.94 .

- Otro error es dejar n fuera de la raíz cuadrada de la fórmula utilizada.

- Y un tercero es considerar el valor 45 en lugar de la proporción 45/200=0.225, con lo cual se obtienen intervalos erróneos.

- Varios alumnos confunden los valores de p, poniendo: 45 o 200/45.

- En cuanto a intervalos hay muchos resultados erróneos.

Cuestión B5:

- Uno de los errores es el valor de z en la tabla ya que dan valores dispares: 5.199; 1.13 o 0.05 .

- Otro es despejar el valor de (sigma), obteniendo resultados que van desde el valor negativo de -93.01, hasta 1960.

De la observación de los errores anteriores encontramos que los alumnos tienen dificultades para cambiar de estrategia, errores que provienen de la dificultad del lenguaje del enunciado, errores que provienen de aplicar reglas equivocadas y sobre todo a la ausencia de comprobación de los resultados obtenidos en el contexto del problema y siguiendo la clasificación de Radatz (1980) -citada por Rico (1995)- podemos afirmar que la mayoría se inscriben en "aprendizaje deficiente de hechos, destrezas y conceptos previos" lo que nos indica que los alumnos han utilizado en la mayoría de los casos recetas y estrategias repetidas una 
y otra vez sin tener presente el razonamiento matemático que sustenta el procedimiento utilizado, que como bien indican González-García, Muñiz-Rodríguez y Rodríguez-Muñiz (2018, p. 449) "revela dificultades causadas por un aprendizaje deficiente de conocimientos previos".

\section{Discusión y Conclusiones}

Los resultados de la opción B son mejores que los de la opción A. En el primer caso se constata que tres cuestiones son fáciles y dos difíciles, mientras que en la opción A hay dos fáciles y tres difíciles. No obstante tres de cada cuatro alumnos han elegido la opción A.

Analizando el tipo de prueba y comparando con casos anteriores (Nortes y Nortes, 2010; Nortes et al., 2015), se observa que en la materia Matemáticas Aplicadas a las Ciencias Sociales II la EBAU es una continuación de la PAU, ya que los contenidos mantienen el mismo esquema por bloques, siguiendo lo indicado en la Orden ECD/42/2018 que desarrolla el Real Decreto 5/2016 "una prueba de características semejantes" (p. 86169) y que viene constatada en la tabla 8:

Tabla 8

Comparativa contenidos de cuestiones PAU-EBAU

\begin{tabular}{cccc}
\hline Cuestión & PAU 2009 & PAU 2014 & EBAU 2018 \\
\hline A1 & Sistema de ecuaciones & Discutir un sistema & Discutir un sistema \\
B1 & Programación lineal & Programación lineal & Programación lineal \\
A2 & Representar una curva & Maximizar función & Maximizar una función \\
B2 & Curva y derivada & Área comprendida & Hallar tres derivadas \\
A3 & Maximizar una función & Hallar dos integrales & Área comprendida \\
B3 & Área comprendida & Hallar dos derivadas & Área comprendida \\
A4 & Probabilidad & Probabilidad de sucesos & Probabilidad condicio- \\
& Teorema de Bayes & Probabilidad condicio- & Probabilidad condicio- \\
B4 & nada & nada \\
A5 & Contraste de hipótesis & Intervalo de confianza & Intervalo de confianza \\
B5 & Intervalo de confianza & Contraste de hipótesis & Intervalo de confianza \\
\hline
\end{tabular}

Comparando los resultados obtenidos con los de estudios anteriores, se mantienen los porcentajes de no superación de esta prueba: en la presente investigación, se obtiene de media 4.32 y en el estudio de Nortes 
De la PAU a la EBAU: un análisis en el dominio de las Matemáticas Aplicadas a las Ciencias Sociales

Rosa Nortes Martínez-Artero, Antonio de Pro Bueno y Andrés Nortes Checa

y Nortes (2010) de 4.5, habiendo sido la media de los 1745 alumnos en 2014 de 4.16 (Nortes et al., 2015), y la media de las muestras de los dos profesores de 3.95 y 4.07.

La resolución del sistema de ecuaciones (Nortes y Nortes, 2010) fue resuelta mayoritariamente por el método de Gauss donde el $85 \%$ lo eligió y aquí lo ha sido por el 66\%. La nota media en PAU 2009 fue de 1.71 frente al 1.21 de EBAU, en una puntuación sobre 3. La cuestión de programación lineal fue resuelta por el $39 \%$ y aquí por el $26 \%$, allí con una media de 0.83 y aquí de 1.73 , con mejores resultados, siendo el porcentaje de aprobados del 55\%, también con la valoración máxima de 3 puntos.

En la cuestión de determinar el área comprendida entre dos curvas en PAU 2009 la media fue de 0.77 y en EBAU de 0.67 en una puntuación sobre 1.5.

Se mantienen los errores en probabilidades y en intervalos de confianza, en esta cuestión en PAU 2009 se obtuvo un porcentaje del 51\% que lo hizo bien y en EBAU en las dos opciones del $42.16 \%$ y $44.44 \%$.

En las cuestiones de probabilidad se siguen dando multitud de respuestas y en algunos casos obteniendo probabilidades superiores a la unidad. Se siguen manteniendo los errores al tomar el valor de las tablas de la distribución normal, operaciones que no finalizan con el valor correcto de lo pedido o el partir mal de los datos. Se mantienen los errores de cálculo y la no comprobación de ver si el resultado tiene sentido en el contexto del problema.

Los resultados en porcentajes en cada estudio vienen en la tabla 9.

Tabla 9

Resultados de las pruebas en porcentajes

\begin{tabular}{cccccc}
\hline & Suspenso & Aprobado & Notable & Sobres. & $\mathrm{N}$ \\
\hline PAU 2009 & 62.7 & 22.4 & 15.4 & 1.5 & 134 \\
PAU 2014. Prof.1 & 70.7 & 14.3 & 12.2 & 2.7 & 147 \\
PAU 2014. Prof.2 & 66.7 & 20.1 & 12.5 & 0.7 & 144 \\
EBAU 2018 & 58.0 & 26.1 & 13.0 & 2.9 & 138 \\
\hline
\end{tabular}

Como características que se mantienen en la EBAU, a modo de resumen citaríamos:

- Contestan mejor aplicando una fórmula que haciendo un razonamiento.

- En los cálculos falta rigor y precisión. 
- Los alumnos siguen siendo entrenados para resolver ejercicios típicos.

- Las pruebas alejan a los docentes del currículo oficial centrándose en el "currículo real".

- Se repite el tipo de problemas a lo largo de los años y no se contextualiza en casos cercanos al alumno.

Como aspecto relevante a considerar en la EBAU se presenta en la Orden PCl/12/2019, en su artículo 5 las matrices de especificaciones que establecen "la concreción de los estándares de aprendizaje evaluables asociados a cada uno de los bloques de contenidos que darán cuerpo al proceso de evaluación" (p. 2679) y que incluye el porcentaje orientativo correspondiente a cada uno de los bloques de contenidos de las materias objeto de evaluación siguiendo lo establecido en el Real Decreto 1105/2014. Según este criterio de las cinco cuestiones de la Opción A, una corresponde al bloque Procesos, métodos y actitudes en matemáticas, dos a Análisis y dos a Estadística y probabilidad, y de las cinco cuestiones de la opción B, una corresponde a Procesos, métodos y actitudes en matemáticas, una a Números y álgebra, una a Análisis y dos a Estadística y probabilidad. Y que además se ajustan a lo establecido en el Decreto 221/2015 de currículo de Bachillerato en la Comunidad Autónoma donde se realiza la prueba.

Como limitaciones del presente estudio está el no poder aportar otros datos complementarios como porqué un alumno elige una opción u otra, el no poder hacer un seguimiento más exhaustivo de la forma de responder a las cuestiones y que el marco teórico solo lo podemos referenciar a la estructura de la prueba. Pero, sobre todo, queremos señalar que la materia Matemáticas Aplicadas a las Ciencias Sociales II (MACS) sigue condicionada por la prueba selectiva de entrada a la universidad, ya que el tipo de cuestiones y el reparto en bloques es semejante en la EBAU y en la PAU. Los errores que se cometen no se han subsanado y los estudios mencionados al inicio de este trabajo siguen siendo válidos en la actualidad. De las 690 cuestiones, el $20 \%$ fueron contestadas bien y el $25 \%$ obtuvieron calificación de cero.

Como rasgo positivo es la disminución del porcentaje de suspensos y aumento de la nota media, con lo que se puede concluir con lo afirmado por Boal et al. (2008) "el estudiante tipo ha sido entrenado para repetir técnicas matemáticas para resolver ejercicios típicos una y otra vez" (p. 
De la PAU a la EBAU: un análisis en el dominio de las Matemáticas Aplicadas a las Ciencias Sociales

Rosa Nortes Martínez-Artero, Antonio de Pro Bueno y Andrés Nortes Checa

22) porque las características de las pruebas y su contenido siguen siendo semejantes aunque se ha delimitado la concreción de los estándares de aprendizaje evaluables asociados a cada uno de los bloques de contenidos.

\section{Referencias}

Boal, N., Bueno, C., Leris, M. D. y Sein-Echaluce, M. L. (2008). Las habilidades matemáticas evaluadas en las pruebas de acceso a la universidad. Un estudio en varias universidades públicas españolas. Revista de Investigación Educativa, 26(1), 11-23.

Decreto 221/2015, de 2 de septiembre de 2015, por el que se establece el currículo de Bachillerato en la Comunidad Autónoma de la Región de Murcia. BORM, 203, 3 de septiembre de 2015, pp. 31594-32533.

González-García, A., Muñiz-Rodríguez, L. y Rodríguez-Muñiz, L.J. (2018). Un estudio exploratorio sobre los errores y las dificultades del alumnado de Bachillerato respecto al concepto de derivada. Aula abierta, 47(4), 449-462.

Lorenzo, M., Argos, J., Hernández, J. y Vera, J. (2014). El acceso y la entrada del estudiante a la universidad: situación y propuestas de mejora facilitadoras del tránsito. Educación XX1, 17(1), 15-38.

Mallart, A. (2014). La resolución de problemas en la prueba de Matemáticas de Acceso a la Universidad: procesos y errores. Educatio siglo XXI, 32(2), 233-254.

Mengual, E., Albarracín, L., Muñoz-Escolano, J. M., Oller-Marcén, A. M. y Gorgorió, N. (2019). Diseño de criterios para reducir la variabilidad en la calificación de exámenes de matemáticas en pruebas de acceso a la universidad. PNA 13(2), 62-83.

Miralles, I. y Deulofeu, J. (2009). Dificultad subjetiva de la prueba de Matemáticas de las PAU. ¿Que eligen los alumnos? XIV Jornadas de Aprendizaje y Enseñanza de las Matemáticas. Girona, España, 1 al 4 de julio.

Nortes, A. y Nortes, R. (2010). Resolución de problemas de Matemáticas en las Pruebas de Acceso a la Universidad. Errores significativos. Educatio Siglo XXI, 28(1), 317-347.

Nortes, R., Nortes, A. y Lozano, F. (2015). Las correcciones en Matemáticas en las Pruebas de Acceso a la Universidad. Educatio Siglo XXI, 33(3), 199-222.

Orden ECD/42/2018, de 25 de enero, por la que se determinan las características, el diseño y el contenido de la evaluación de Bachillerato para el acceso a la Universidad, las fechas máximas de realización y de resolución de los procedimientos de revisión de las calificaciones obtenidas, para el curso 2017/2018. Boletín Oficial del Estado, 23, 26 de enero de 2018, pp. 9757-9789.

Orden $\mathrm{PCl} / 12 / 2019$, de 14 de enero, por la que se determinan las características, el diseño y el contenido de la evaluación de Bachillerato para el acceso a la Universidad, las fechas máximas de realización y de resolución de los procedimientos de revisión de las calificaciones obtenidas, para el curso 2018/2019. Boletín Oficial del Estado, 13, 15 de enero de 2019, pp. 2677-2728. 
De la PAU a la EBAU: un análisis en el dominio de las Matemáticas Aplicadas a las

Ciencias Sociales

Rosa Nortes Martínez-Artero, Antonio de Pro Bueno y Andrés Nortes Checa

Ordóñez, L. y Contreras, A. (2011). La integral definida en bachillerato. Restricciones institucionales de las Pruebas de Acceso a la Universidad. En M. Marín, G. Fernández, L. Blanco y M. Palarea (Eds.), Investigación en Educación Matemática XV, (pp. 461470). Ciudad Real: SEIEM.

Real Decreto 1105/2014, de 26 de diciembre, por el que se establece el currículo básico de la Educación Secundaria Obligatoria y del Bachillerato. Boletín Oficial del Estado, 3, 3 de enero de 2015, pp. 169-546.

Real Decreto-ley 5/2016, de 9 de diciembre, de medidas urgentes para la ampliación del calendario de implantación de la Ley Orgánica 8/2013, de 9 de diciembre, para la mejora de la calidad educativa. Boletín Oficial del Estado, 298, 10 de diciembre de 2016, pp. 86168-86174.

Rico, L. (1995): Errores y dificultades en el aprendizaje de las Matemáticas. En J. Kilpatrik, P. Gómez y L. Rico (Coords.), Educación Matemática (pp. 69-108). Méjico: Grupo Editorial Iberoamérica.

Rodríguez-Muñiz, L.J., Torres, E. y Álvarez-Martino, E. (2012). Diseño metodológico para la elaboración de estadísticas de la Prueba de Acceso a la Universidad. Aula Abierta, 40(3), 83-96.

Rodríguez-Muñiz, L. J., Díaz, P., Mier, V. \& Alonso, P. (2016). Washback Effect of University Entrance exams in Applied Mathematics to Social Sciences. PLoS ONE 11(12), 1-18: e0167544. doi:10.1371/journal.pone.0167544.

Ruíz de Gauna, J. (2010). La enseñanza de las matemáticas del bachillerato, los libros de texto y las pruebas de acceso a la UPV/EHU (1970-2008). (Tesis doctoral, Universidad del País Vasco, España). Recuperada de http://hdl.handle.net/10810/12419

Ruíz de Gauna, J., Dávila, P., Etxeberría, J. y Sarasua, J. (2013). Pruebas de selectividad en Matemáticas en la UPV-EHU. Resultados y opiniones del Profesorado. Revista de Educación, 362, 217-246. 
